\title{
QUALIDADE NUTRICIONAL E PARÂMETROS MORFOLÓGICOS DO FRUTO CAJÁ (SPONDIAS MOMBIN L.)
}

Nutritional-functional quality and morphological parameters of fruit Cajá (Spondias Mombin L.)

Calidad nutricional-funcional y parámetros morfológico del fruto caja (Spondias Mombin L.)

Rayssa Caroline de Almeida Silva Silvino1, Graziela Cristina Teles da Silva2, Orquídea Vasconcelos dos Santos 1*

${ }^{1}$ Laboratório de Ciências dos Alimentos, Curso de Nutrição, Universidade Federal do Pará, Belém-PA, Brasil.

${ }^{2}$ Faculdade de Física, Universidade Federal do Pará, Belém-PA, Brasil.

*Correspondência: Laboratório de Ciência dos Alimentos, Universidade Federal do Pará, Cidade Universitária Prof. José da Silveira Neto - Campus Profissional III - Complexo Saúde, Rua Augusto Corrêa, 01, Guamá, Belém, Pará, Brasil.CEP: 66075-110.e-mail orquideavs@ufpa.br

\section{Artigo recebido em 21/01/2017 Aprovado em 29/03/2017 Publicado em 19/04/2017.}

\section{RESUMO}

O aumento no consumo e na produção de frutas tropicais promove a necessidade de pesquisas direcionadas a novas vertentes de compostos Nutricionais e funcionais da biodiversidade Amazônica. Assim, objetivou-se nesta pesquisa analisar a qualidade e o potencial funcional da spondias mombin L., bem como seu padrão morfológico e o perfil espectroscópico. As bases metodológicas e métodos aplicados seguiram o padrão de análise internacionalmente aceitas indicados pela Association of Official Analytical Chemists (AOAC) e Legislação Brasileira, para as análises morfológicas utilizou-se a Microscopia Eletrônica de Varredura. A partir dos resultados obtidos evidenciou-se um fruto de considerável valor nutricional com baixo valor energético e alta relevância em compostos funcionais como a vitamina $\mathrm{C}$, com médias de 25,37 mg ácido ascórbico/100 g e teor de carotenoides totais com valor médio de 1498,57 ( $\mu \mathrm{g} .100 \mathrm{~g}^{-1}$ ) evidenciando-se a relevância desse fruto em provitamina A. As micrografias das estruturas revelaram uma matriz vegetal com presença de fibras e de raros grânulos de amido. A presença de fibras e das vitaminas $\mathrm{A}$ e $\mathrm{C}$ nesta composição aliado com seu baixo valor energético mostrou a capacidade de prevenção de determinadas patologias que o consumo deste fruto pode oferecer.

Palavras-Chave: Cajá; vitaminas; micrografia.

\section{ABSTRACT}

The increase in consumption and production of tropical fruits promotes the need for research aimed at new aspects of nutritional and functional compounds of the Amazonian biodiversity. Thus, the objective of this research was to analyze the quality and functional potential of spondias mombin L., as well as its morphological pattern and the spectroscopic profile. The methodological bases and methods applied followed the internationally accepted standard of analysis indicated by the Association of Official Analytical Chemists (AOAC) and Brazilian Legislation, for the morphological analysis was used Scanning Electron Microscopy. From the obtained results, a fruit of considerable nutritional value with low energy value and high relevance in functional compounds such as vitamin C, with averages of $25.37 \mathrm{mg}$ ascorbic acid $/ 100 \mathrm{~g}$ and its total carotenoid content with an average value of 1498,57 ( $\left.\mu \mathrm{g} .100 \mathrm{~g}^{-1}\right)$ showing the relevance of this fruit in pro-vitamin A. Micrographs of the structures revealed a plant matrix with presence of fibers and rare starch granules. The presence of fibers and vitamins $A$ and $C$ in this 
composition combined with its low energy value showed the ability to prevent certain pathologies that the consumption of this fruit can offer.

Keywords: Cajá; vitamins; micrography.

\section{RESUMEN}

El aumento en el consumo y la producción de frutas tropicales promueve la necesidad de una investigación dirigida a nuevos aspectos de compuestos nutricionales y funcionales de la biodiversidad de la Amazonia. Por lo tanto, este estudio tuvo como objetivo evaluar la calidad y funcional potencial de Spondias mombin L., Así como su morfología y el perfil espectroscópico. Las bases metodológicas y aplican métodos seguidos análisis norma internacionalmente aceptada que se indica por la Asociación de Químicos Analíticos Oficiales (AOAC) y la legislación brasileña, para el análisis morfológico utilizó la microscopía electrónica de barrido. De los resultados se desprende un considerable valor nutricional de frutas con bajo consumo de energía y de alta importancia en compuestos funcionales, tales como la vitamina C, con un promedio de 25,37 mg de ácido ascórbico / $100 \mathrm{~g}$ y su contenido total de carotenoides con un promedio de $1498.57\left(\mu \mathrm{g} .100 \mathrm{~g}^{-1}\right)$ para mostrar la relevancia de esta fruta en provitamina A. las micrografías de las estructuras reveló una matriz vegetal con la presencia de fibras y granos raros de almidón. La presencia de la fibra y las vitaminas $A$ y $C$ en esta composición junto con su bajo valor energético mostró la capacidad de prevenir ciertas enfermedades que el consumo de esta fruta puede proporcionar.

Descriptores: Caja; vitaminas; micrografía.

\section{INTRODUÇÃO}

A família Anacardiaceae engloba diversas espécies. Esta possui aproximadamente 81 gêneros e 800 espécies. No Brasil, estão catalogados 14 gêneros com 57 espécies, sendo 14 espécies endêmicas (Silva-Luz; Pirani, 2010). Entre as espécies frutíferas importantes destacam-se as do gênero Spondias (cajá, cajarana, umbu-cajá, seriguela). A exploração dessas espécies ocorre de forma extrativista, a grande maioria do cultivo se dá em pomares domésticos, ficando as agroindústrias dependentes dessa produção, que é sazonal, mas apesar de não estar incluídas em dados oficiais possuem grande importância socioeconômica para as regiões Norte e Nordeste do Brasil, nesses locais são comercializados em feiras livres e às margens de estradas. (Pinto et al., 2003; Souza, 2005).

$\mathrm{Na}$ Amazônia o fruto da cajazeira é conhecido como Taperebá e no nordeste como Cajá, esses nomes fazem referência às palavras tapiriba e acaja, como eram chamadas pelos indígenas (Carvalho et al., 2011). De formato ovoide, possui mesocarpo pouco carnoso, amarelo alaranjado quando maduros, tendo um sabor agridoce. O fruto é extremamente aromático, sendo essa característica responsável, em parte, pela sua boa aceitabilidade (Bosco et al., 2000; Soares, 2005). O comprimento e o peso do fruto são considerados fatores importantes na escolha de frutos destinados as indústrias, como a de sucos (Pinto et al., 2003).

O cajá tem um aspecto funcional relevante, pois possui elevado teor de carotenoides, vitamina $\mathrm{C}$ e taninos, que podem atuar como substâncias antioxidantes colaborando para o aumento do consumo e interesse das indústrias alimentícias que o utiliza como matéria-prima (Mattietto et al., 2010). Além disso, Alguns estudos têm demonstrado que não só o fruto pode ter um valor econômico de destaque, mas também as folhas e a casca da árvore, que podem ser usadas no tratamento de pé-de-atleta, por exemplo. Com isso é revelado algumas potencialidades medicinais da árvore e não apenas como uma árvore agroflorestal (Adedokun et al., 2010).

Esta pesquisa tem como objetivo analisar a qualidade nutricional e o potencial funcional do fruto 
cajá (spondias mombin L.), bem como seu padrão morfológico e o perfil espectroscópico.

\section{MATERIAIS E MÉTODOS}

\section{PREPARAÇÃO DA AMOSTRA}

Foram adquiridos $10 \mathrm{~kg}$ do fruto do cajá in natura, provenientes do comercio de fruticultura do município de Belém, referentes à safra de 2015. As amostras foram transportadas em sacos plásticos de Polietileno de Baixa Densidade (PEBD) sendo armazenadas no Laboratório de Análise de alimentos na UFPA à temperatura de $7{ }^{\circ} \mathrm{C}$. Houve a execução dos procedimentos de recepção, seleção, higienização, lavagem, corte e secagem. $\mathrm{O}$ processo de secagem foi realizado em estufa de circulação de ar forçado em temperatura de $55^{\circ} \mathrm{C}$ por $24 \mathrm{~h}$, obtendo-se material seco que foi posteriormente triturado em moinho de facas tipo Willye marca TECNAL modelo TE650.

\section{ANÁLISE MICROBIOLÓGICA}

As amostras do fruto do cajá in natura foram analisadas de forma indicativa, sendo submetidas às análises microbiológicas segundo parâmetros de tolerância exigidos pela Resolução RDC no 12 , de 02 de janeiro de 2001 (BRASIL, 2001), e de acordo com metodologias descritas por Vanderzant e Splittstoesser (1992).

\section{CARACTERIZAÇÃO FÍSICA E FÍSICO- QUÍMICA}

A biometria do fruto foi realizada após coleta, seleção e higienização do fruto. Para mensuração do diâmetro maior e menor, utilizou-se um paquímetro (VONDER) e, para os pesos, uma balança analítica da marca QUIMIS (Electronic Balance FA-2104n, Bioprecisa).

Atividade de água: através de medida direta, em instrumento AquaLab Series 3TE da DECAGON, com controle interno de temperatura a $25^{\circ} \mathrm{C}$;

$\mathrm{pH}$ : realizado segundo o método $\mathrm{n}^{\circ} 981.12$ da AOAC (2000), por meio do uso de um potenciômetro, previamente calibrado com soluções tampão pH 4 e 7;

Acidez Total Titulável: realizada segundo o método $\mathrm{n}^{\circ} 22.058$ da AOAC (2000) que mede a acidez titulável das substâncias;

Sólidos solúveis Totais: realizada segundo método IAL (1985) por refratômetria, com o uso de refratômetro manual de marca Tecnal, modelo AR200 digital.

Índice Ratio: calculado através da relação entre sólidos solúveis totais e Ac. titulável, segundo Reed et al. (1986).

\section{COMPOSIÇÃO NUTRICIONAL BASEADA NAS ANÁLISES FÍSICO-QUÍMICAS}

Umidade: Realizada de acordo com o método 920.151 da Association of Official Analytical Chemists AOAC (2000).

Resíduo mineral fixo (cinzas): Realizada por calcinação, de acordo com o método AOAC (2010).

Proteína: Realizada pelo método de Kjeldahl, de acordo com o método AOAC (2010).

Lipídeos: Realizada por extração com éter de petróleo empregando extrator Soxhlet, de acordo com o método AOAC (2010).

Fibras brutas: Determinada pelo método detergente ácido (ADF) Van Soest (1967);

Carboidratos: Realizada por diferença, segundo a Resolução RDC $n^{\circ} 360$, de 23 de dezembro de 2003 (Brasil, 2003). 
Valor calórico: Realizada aplicando os fatores de Awater 4 - 9 - 4 kcal/g, para os valores de proteínas, lipídeos e carboidratos, respectivamente, de acordo com a Resolução RDC n ${ }^{\circ} 360$, de 23 de dezembro de 2003 (Brasil, 2003).

\section{COMPOSIÇÃO NUTRICIONAL: ANÁLISE DE CONCENTRAÇÃO DE CAROTENOIDES E DE VITAMINA C}

Análise de Concentração de Carotenóides: realizada por leitura espectroscópica em absorbância de $450 \mathrm{~nm}$ conforme as metodologias descritas por França e Meireles (2000).

Análise da Concentração de Vitamina C: realizada a partir da metodologia titulométrica aplicada seguiu método oficial proposto pela AOAC 43.065 (1997).

\section{ANÁLISE MORFOLÓGICA}

As análises morfológicas do cajá foram realizadas pela visualização em Microscopia Eletrônica de Varredura (MEV), com amostras previamente secas em estufas de circulação de ar a $105{ }^{\circ} \mathrm{C}$ por 48 horas, posteriormente acopladas em suportes e metalizados com ouro com uma camada aproximada de $20 \mathrm{~nm}$ de espessura por 150 segundos em corrente de $25 \mathrm{~mA}$ (Silveira, 1989). As imagens ou eletromicrografias foram realizadas em Microscópio eletrônico de varredura marca LEO modelo $1450 \mathrm{VP}$.

\section{RESULTADOS E DISCUSSÃO}

$\mathrm{Na}$ caracterização física, foi realizada a análise biométrica. Essa análise é fundamental, pois o peso e comprimento do fruto são considerados fatores importantes na escolha dos frutos destinados à indústria (Tabela 1).
Tabela 1. Parâmetros biométricos do fruto cajá (Spondias Mombin L.).

\begin{tabular}{lccc}
\hline & Mín. & Máx. & Média \\
\hline Comprimento (cm) & 2,18 & 3,53 & $2,89 \pm 0,26$ \\
Largura (cm) & 1,83 & 2,81 & $2,27 \pm 0,20$ \\
Peso inteiro (g) & 4,75 & 13,7 & $8,56 \pm 2,02$ \\
\hline
\end{tabular}

Dados expressam a média \pm desvio-padrão de 100 frutos

Os resultados da caracterização física do cajá indicaram uma considerável variabilidade. Para Bosco et al. (2000) são considerados frutos grandes aqueles que pesam mais de $15 \mathrm{~g}$; médios se pesarem entre 12 e $15 \mathrm{~g}$; pequenos os que tiverem valores inferiores a 12 g. O peso dos frutos no presente estudo variou bastante, de 4,75 a 13,73 g. No trabalho de Carvalho et al. (2011) também houve grande variação em relação ao peso, sendo encontrados valores de 6,15 a 18,82 g, assim como os valores apresentados por Soares et al. (2006), em que a variação foi de 5,7 a 16,6 g. O peso do fruto é uma variável bastante importante para as indústrias, pois quanto maior o peso, maior a atração do consumidor e maior a rentabilidade das indústrias. Em relação ao comprimento, Carvalho et al. (2011) encontrou uma variação de 2,64 a 3,38 cm, e Soares et al. (2006), uma variação de 2,95 a 3,98 cm, valores próximos aos relatados neste estudo. Para a largura, Mattietto et al. (2010) obteve uma variação de 1,33 a 2,73 cm, valores estes bem semelhantes aos achados neste trabalho, assim como os relatados por Soares et al. (2006), que foram de 1,83 a 2,68.

Também fez parte das análises a medição dos seguintes parâmetros: $\mathrm{pH}$, atividade de água, sólidos solúveis, acidez total titulável e índice Ratio (Tabela 2).

Tabela 2. Composição física do fruto cajá. Análises Resultados* 
$\mathrm{pH}$

Atividade de água

$1,45 \pm 0,07$

Acidez titulável (\% ácido cítrico)

$0,99 \pm 0,01$

Sólidos solúveis ( ${ }^{\circ}$ Brix)

$1,69 \pm 0,21$

Índice Ratio

$10,00 \pm 0,04$

$5,92 \pm 0,07$

*Dados expressam a média das triplicatas \pm desvio-padrão

Diante dos resultados obtidos nas análises físico-químicas, é possível perceber que se trata de um fruto de polpa bastante ácida, apresentando $\mathrm{pH}$ de 1,45 . De acordo com a Instrução Normativa $n^{\circ}$ 01, de 7 de janeiro de 2000 (Brasil, 2000), o pH mínimo para a polpa de cajá é de 2,2, o que diverge do achado no presente trabalho. A acidez titulável de $1,69 \%$ encontra-se em conformidade com a legislação vigente, que estabelece o mínimo de 0,90\% para acidez titulável total expressa em ácido cítrico. Esse resultado assemelha-se aos obtidos nos trabalhos de Mattietto et al. (2010) que encontrou uma acidez titulável total de 1,86\% e Carvalho et al. (2011), que obteve resultados que variaram de $1,20 \%$ a $1,93 \%$, ambos os trabalhos realizados com o cajá. Os resultados encontrados para açúcares totais $\left(4,32 \mathrm{~g} .100 \mathrm{~g}^{-1}\right)$ e açúcares redutores $(4,19 \mathrm{~g}$. $100 \mathrm{~g}^{-1}$ ) foram semelhantes aos obtidos no estudo de Mattietto et al. (2010) e bem abaixo dos valores encontrados nos trabalhos de Pinto et al. (2003) e Dias et al. (2003), porém enquadram-se na exigência da Instrução Normativa $n^{\circ}$ 01, que é de no máximo $12 \mathrm{~g} / 100 \mathrm{~g}$ para açúcares totais. De acordo com a legislação existente em relação aos teores de sólidos solúveis para a polpa de cajá, o mínimo é de 9,0 ${ }^{\circ}$ Brix; sendo assim, os resultados obtidos estão dentro do exigido, já que foi encontrado o valor de $10,0{ }^{\circ}$ Brix, valor este próximo ao encontrado nos trabalhos de Soares et al. (2006) e Mattietto et al. (2010).

A relação expressa pelo índice Ratio mostra o grau de maturação médio do fruto do cajá.
O sabor dos frutos é dependente direto das quantidades de açúcares (sólidos solúveis totais ${ }^{\circ}$ Brix) e da acidez total titulável. Não há uma escala de valores definidos para esse índice, sendo dependente do tipo de fruta a ser processado e/ou consumido in natura e do seu respectivo grau de maturação.

Para a pesquisa microbiológica foram feitas as seguintes análises: coliformes a $45^{\circ}$ C e Salmonella sp. (Tabela 3).

Tabela 3. Resultados da análise microbiológica de amostras da polpa in natura do fruto cajá.

Análise Resultados

Microbiológica

\begin{tabular}{ll}
\hline Escherichia coli & $<3 \mathrm{NMP} \cdot \mathrm{g}^{-1}$ \\
Estaf. coag. positiva & $<1 \times 10^{-1} \mathrm{UFC} \cdot \mathrm{g}^{-1}$ (ausente) \\
Salmonella sp. & Ausente \\
Bolores e Leveduras & $<1,5 \times 10^{2} \mathrm{UFC} \cdot \mathrm{g}^{-1}$
\end{tabular}

análise microbiológica realizada na polpa de cajá revelou-se em conformidade com os padrões exigidos na legislação vigente. Não foram detectadas contaminação por Estaf. coag. positiva ou Salmonella sp., que de acordo com o padrão exigido na Resolução RDC $\mathrm{n}^{\mathrm{o}}$ 12, de 02 de janeiro de 2001, da Agência Nacional de Vigilância Sanitária (ANVISA), estabelece ausência de Salmonella sp em 25 g. Nas análises realizadas por Dantas et al. (2012) com polpas de frutas, entre elas a polpa de cajá, foi obtido resultado semelhante, assim como no trabalho de Nascimento et al. (2006), em que $100 \%$ das amostras obtiveram ausência de Salmonella sp.. Em relação aos coliformes à $45^{\circ}$, do qual parte significante refere-se a Escherichia coli, também foi encontrado resultado (< 3NMP.g $\left.{ }^{-1}\right)$, de acordo com a legislação, que estabelece o padrão máximo de $100 \mathrm{NMP} \cdot \mathrm{g}^{-1}$. Nos estudos de Nascimento et al. (2006), Filho et al. (2010) e Santos et al. (2008) com amostras de polpas de frutas, foram 
obtidos resultados parecidos, o que reflete adequadas condições higiênico-sanitárias no processamento. Em relação aos teores de bolores e leveduras, a Instrução Normativa do MAPA (Brasil, 2000) estabelece como padrão para a polpa in natura a soma de máximo $5 \times 10^{3}$ UFC. $g^{-1}$. O resultado enquadra-se na legislação, diferentemente do encontrado por Nascimento et al. (2006) em que todas as suas amostras estavam contaminadas refletindo sério perigo de saúde pública, pois estes microrganismos produzem toxinas nocivas a saúde do ser humano. Da mesma forma, no trabalho de Santos et al. (2008), 88 amostras (89,8\%) apresentaram valores acima do limite máximo preconizado para bolores e leveduras, resultados semelhantes também foram encontrados nos trabalhos de Sebastiany et al. (2009), que avaliou polpas comercializadas em Roraima, e de Leite et al. (2000), que analisou polpas produzidas na Bahia.

A composição nutricional do fruto foi determinada, bem como foram realizadas análises da sua composição funcional (Tabela 4).

Tabela 4. Caracterização nutricional e funcional do

\begin{tabular}{|c|c|}
\hline Composição centesimal/100 g & Resultados* \\
\hline Valor energético (kcal) & 49,15 \\
\hline Umidade (\%) & $86,78 \pm 0,38$ \\
\hline Cinzas (\%) & $2,62 \pm 0,51$ \\
\hline Lipídios totais (\%) & $1,35 \pm 0,16$ \\
\hline Proteína (\%) & $2,93 \pm 0,00$ \\
\hline Carboidratos totais (g) & $6,32 \pm 0,00$ \\
\hline Açúcares totais (g. $\left.100 \mathrm{~g}^{-1}\right)$ & $4,32 \pm 0,15$ \\
\hline Açúcares redutores $\left(\mathrm{g} .100 \mathrm{~g}^{-1}\right)$ & $4,19 \pm 0,06$ \\
\hline Fibras insolúveis (\%)** & $1,95 \pm 0,78$ \\
\hline Vitamina C (mg ác. Ascórbico.100g $\left.{ }^{-1}\right)^{* *}$ & $25,37 \pm 0,63$ \\
\hline Carotenoides Totais $\left(\mu \mathrm{g} .100 \mathrm{~g}^{-1}\right) * *$ & $1498,57 \pm 0,02$ \\
\hline
\end{tabular}

Em relação à composição centesimal, é possível perceber que é um fruto com alto teor de água $(86,78 \%)$, baixo teor de lipídios e proteínas. Os resultados encontrados no presente trabalho encontram-se próximos aos estudos de Mattietto et al.
(2010). A pesquisa de Lago-Vanzela et al. (2011) obtive na análise de lipídios da polpa de cajá o resultado de $0,85 \%$, valor este abaixo dos achados nesse estudo. Para proteínas Lago-Vanzela et al. (2011) obteve 1,47\%, divergindo do resultado encontrado neste trabalho, que foi de $2,93 \%$. No trabalho realizado por Tiburski et al. (2011), analisando as propriedades nutricionais da polpa de cajá foram encontrados $0,62 \%$ de lipídios, $1,06 \%$ de proteínas e 13,90\% de carboidratos. Estes dados mostram as diferenças entre as variedades de frutos, safras, localizações, entre outros aspectos.

O teor de fibras obtido nesta pesquisa (em média de $1,95 \%$ ) mostra a relevância funcional da composição deste fruto no que tange a prevenção de agravos patológicos e sua ação em termos tecnológicos na possibilidade da aplicação desta em conjunto com outras matrizes alimentares.

O teor de vitamina $\mathrm{C}$ e a quantificação de carotenoides totais também foram determinados. O teor de carotenoides obtido neste trabalho foi de 1498,57 $\mu \mathrm{g} .100 \mathrm{~g}^{-1}$, resultados próximo ao do estudo de Carvalho et al. (2011) com amostras de cajazeiras, que obteve resultados que variaram de 1070,00 a $3760,00 \mu \mathrm{g} .100 \mathrm{~g}^{-1}$ de carotenoides. Sobrinho (2014) encontrou em seu estudo, com amostras provenientes de um pomar na Bahia, o valor médio de 2580,00 $\mu \mathrm{g} .100 \mathrm{~g}^{-1}$, que difere um pouco do encontrado no presente estudo. Essas variações nos teores de carotenoides se devem a diversos fatores, como o clima, a localização geográfica de produção, as condições de plantio e também o estágio de maturação na colheita (Rodriguez-Amaya; Kimura, 2008). Em relação à Vitamina $\mathrm{C}$, devido ao seu alto poder redutor, esta atua como potente antioxidante inativando os radicais livres, nutrindo as células e protegendo-as contra danos oxidativos, ajudando também na imunidade, na cicatrização de feridas e na 
prevenção de doenças degenerativas. O resultado obtido nesta pesquisa foi em média de $25,37 \mathrm{mg}$ ácido ascórbico/100 g cajá, valor próximo ao encontrado nas pesquisas de Mattieto et al. (2010), com médias de 23,72 mg ácido ascórbico/100g.

A caracterização morfológica do fruto também foi analisada, pois o estudo da sua estrutura através da Microscopia Eletrônica de Varredura (MEV) é fundamental para o estudo da sua capacidade funcional (Figuras 1e 2).

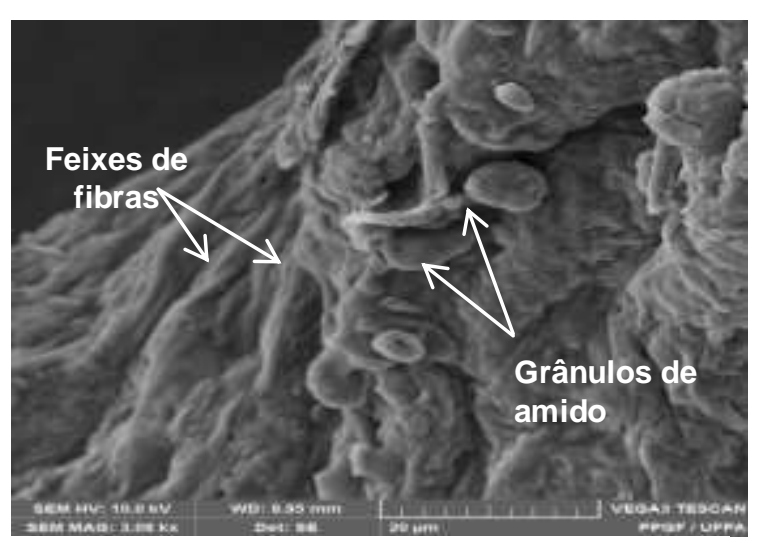

Figura 1. Estrutura da Matriz vegetal

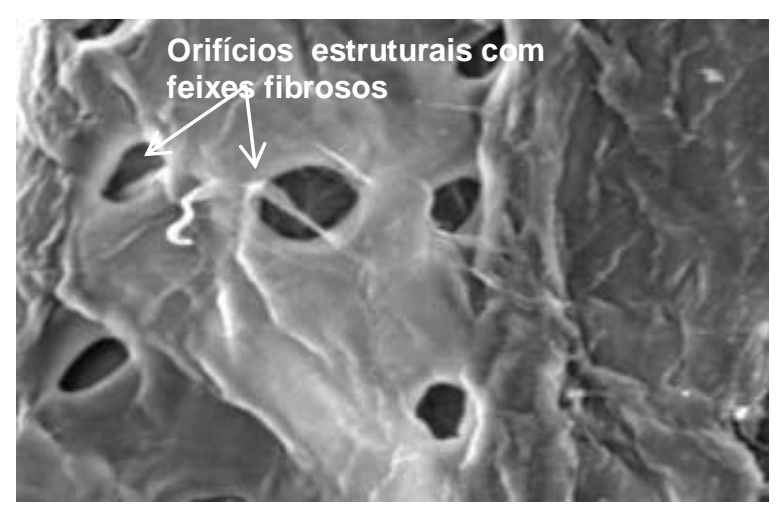

Figura 2. Estrutura de Membrana da matriz vegetal

Em relação à Microscopia Eletrônica de Varredura da matriz vegetal, é possível verificar a ratificação de aspectos funcionais em feixes de fibras. Essa característica demonstra mais um efeito benéfico funcional do consumo deste fruto, pois as fibras ajudam no funcionamento regular do intestino, contribuindo para a eliminação de toxinas. Além disso, as frutas ricas em fibras ajudam na sensação de saciedade e isso pode ajudar no processo de perda de peso, bem como na redução de algumas Doenças Crônicas Não Transmissíveis (DCNT), como diabetes e alguns tipos de câncer (Pimentel, 2005).

Na Figura 2 é possível visualizar a superfície de membrana do fruto cajá composta por numerosos poros entremeados de feixes fibrosos ratificando suas propriedades tecnológicas aliadas a sua funcionalidade. A capacidade de absorção de água, óleo e demais materiais solúveis oriundos de misturas para a produção de novas formulações se ampara na capacidade de formação de "corpo" para a estruturação de uma massa homogênea a partir da característica esponjosa do material, observadas por suas respectivas estruturações de matrizes esponjosas.

\section{CONCLUSÃO}

Os resultados demonstraram que o fruto cajá (Spondias mombin L.) é um alimento que possui alto teor nutricional e funcional, em virtude de seus baixos teores energéticos e da presença de fibras, aliados a consideráveis valores de vitamina $\mathrm{C}$ e de provitamina A, expressa em seus teores de carotenoides totais. Portanto, trata-se de um alimento fonte de vitamina $\mathrm{C}$ e possui alto conteúdo de provitamina A.

As análises microbiológicas apresentaram resultados adequados, de acordo com os parâmetros estabelecidos pela legislação.

As análises físico-químicas revelam um fruto com alta atividade de água, de polpa bastante ácida e com um índice de maturação relativamente baixo.

As estruturas de membrana celular mostradas como predominância nesta matriz vegetal ratificam o seu baixo valor energético, pela pouca ocorrência de amidos, em sua estrutura e considerável presença de fibras insolúveis. 
Os parâmetros morfológicos e a composição físico-química do cajá demostraram as potencialidades nutricionais e funcionais ratificando suas respectivas constituições e ações no sistema metabólico de defesa orgânica, atuando na prevenção de agravos como potente antioxidante orgânico. Assim, seu consumo regular agrega diversos benefícios para a saúde, como a melhora da imunidade, do funcionamento intestinal e a prevenção de alguns tipos de patologias crônicos degenerativos.

Todos os autores declararam não haver qualquer potencial conflito de interesses referente a este artigo.

\section{REFERÊNCIAS}

ADEDOKUN, M.O.; OLADOYE, A.O.; OLUWALANA, A.S.; MENDIE, I.I. Socio-economic importance and utilization of Spondias mombin in Nigeria. Asian Pacific Journal of Tropical Medicine 3: 232-234, 2010.

AMORIM, J. A. 1999. Caracterização de alguns bagaços de polpa de frutas obtidos na indústria de processamento Milfrutas indústria alimentícias LTDA. Relatório de Estágio Supervisionado do Departamento de Engenharia Química da UFRN. Natal - RN.

ASSOCIATION OF OFFICIAL ANALYTICAL CHEMISTS - AOAC. Official methods of analysis of the AOAC. Washington, 1997. p. 16-17. (v. 2)

ASSOCIATION OF OFFICIAL ANALYTICAL CHEMISTS. Official Methods of Analysis. 16. ed., Virginia, 2010.

BOSCO, J.; SOARES, K.T.; AGUIAR FILHO, S.P. de.; BARROS, R.V. A. 2000. cultura da cajazeira. João Pessoa: EMEPA.

BRASIL. 2001. Leis, decretos, etc. Resolução RDC n. 12, de 02 de janeiro de 2001. Aprova o regulamento técnico sobre padrões microbiológicos para alimentos. Diário Oficial da República Federativa do Brasil, Brasília, DF, 10 de janeiro de 2001, seção 1, p. 45-53.

BRASIL. 2000. Leis, Decretos, etc. Instrução Normativa $\mathrm{n}^{\circ} 1$, de 7 jan. 2000, do Ministério da Agricultura. Diário Oficial da União, Brasília, n. 6,
10 jan. 2000. Seção I, p. 54-58. Aprova os Regulamentos Técnicos para fixação dos padrões de identidade e qualidade para polpas e sucos de frutas.

BRASIL. 2003. Ministério da saúde. Agência Nacional de Vigilância Sanitária (ANVISA), Diário Oficial da União Resolução RDC $n^{\circ} 360$. Regulamento técnico sobre rotulagem nutricional de alimentos embalados Brasília, DF. Dezembro de 2003.

CARVALHO, A. V.; CAVALCANTE, M. A.; SANTANA, C. L. ALVES, R. M. Características físicas, químicas e atividade antioxidante de frutos de matrizes de cajazeira no estado do Pará. Alimentos e Nutrição Araraquara, v.22, n.1, p. 45-53, jan/mar. 2011.

CUNHA SOBRINHO, A. P.; MAGALHÃES, A. F. DE. J.; SOUZA, A. DA S.; PASSOS, O. S.; FILHO, W. DOS S. S. (Ed.). Cultura dos citros. Vol. 1. Brasília, DF: Embrapa, 2014.

DANTAS, R. L.; ROCHA, A. P. T.; ARAÚJO, A. S.; RODRIGUES, M. S. A.; MARANHÃO, T. K. L. Qualidade microbiológica de polpa de frutas comercializadas na cidade de Campina Grande/PB. Revista Brasileira de Produtos Agroindustriais, Campina Grande, v.14, n.2, p.125-130, 2012.

DIAS, D. R.; SCHWAN, R. F.; LIMA, L. C. O. Metodologia para elaboração de fermentado de cajá. Ciência e Tecnologia de Alimententos, Campinas, 23(3): 342-350, set.-dez.2003.

FILHO, L. F. Q. T.; GODOY, R. C. B.; TESHIMA, E.; CARDOSO, R. L.; BARBOSA, P. R. S.; SANTANA, D. N. R. Avaliação microbiológica da polpa de cajá conservada por métodos combinados. Revista do Instituto Adolfo Lutz, v, 69, n. 4, p. 510517, 2010.

FRANÇA, F. L.; MEIRELES, M.A. Modeling the extraction of carotene and lipids from pressed palm oil (Elaes guineensis) fibers using supercritical $\mathrm{CO} 2$. Journal of Supercritical Fluids, v.18, p. 35-47, 2000.

INSTITUTO ADOLFO LUTZ. Métodos químicos para análise de alimentos. 3. ed. São Paulo, 1985.

LAGO-VANZELA, E. S.; RAMIN, P.; UMSZAGUEZ, M. A.; SANTOS, G. V.; GOMES, E.; SILVA, R. Caracterização química e sensorial de geléia da casca e polpa de cajá-manga (Spondias cytherea Sonn.). Ciência e Tecnologia de Alimentos, v. 31, p. 398- 405, 2011. 
LEITE, C. C.; SANTANA, L. R. R.; SILVA, M. D.; SANT'ANNA, M. E. B.; ASSIS, P. N. Avaliação Microbiológica de polpas congeladas de frutas produzidas no Estado da Bahia. Higiene Alimentar, v. 11, n. 78-79, p. 69-73, 2000.

MATTIETTO, R. A.; LOPES, A. S.; MENEZES, H. C.; Caracterização física e físico-química dos frutos da cajazeira (Spondias mombin L.) e de suas polpas obtidas por dois tipos de extrator. Brazilian Journal of Food Technology, v.13, n.3, p. 156-164, jul./set. 2010 .

NASCIMENTO, A.R.; FILHO, J.E.M.; MARINHO, S. C.; MARTINS, A. G. L. A.; SOUZA, M. R.; SILVA, W. A. S. S.; CASTILLO, F. A.; OLIVEIRA, M. B. Incidência de microrganismos contaminantes em polpas de frutas comercializadas in natura em feiras livres da cidade de São Luís/MA. B. CEPPA, Curitiba, v. 24, n. 1, p. 249-258, 2006.

PIMENTEL, B. M. V.; FRANCKI, M.; GOLLÜCKE, B. P. Alimentos funcionais: introdução as principais substâncias bioativas em alimentos. São Paulo, Varella, 2005. 95p.

PINTO, W. S.; DANTAS, A. C. V. L.; FONSECA, A. A. O.; LEDO, C. A. S.; JESUS, S. C.; CALAFANGE, P. L. P.; ANDRADE, E. M. Caracterização física, físico-química e química de frutos de genótipos de cajazeiras. Pesquisa agropecuária brasileira, Brasília, v. 38, n. 9, p. 1059-1066, set. 2003.

REED, J.B.; HENDRIX, D.L.; HENDRIX JR., C.M. 1986. Quality control manual for citrus processing plants. Safety Harbor: Intercit, v.1.

RODRIGUEZ-AMAYA, D. B.; KIMURA, M. 2008. Fontes brasileiras de carotenóides: tabela brasileira de composição de carotenoides em alimentos. MMA/SBF: Brasília - DF, 100p.

SANTOS, C.A.A.; COELHO, A. F. S.; CARREIRO, S. C. Avaliação microbiológica de polpas de frutas congeladas. Ciência e Tecnologia de Alimentos, v. 4, p.913-915, 2008.

SEBASTIANY, E.; REGO, E. R.; VITAL, M. J. S. Qualidade microbiológica de polpas de frutas congeladas. Revista do Instituto Adolfo Lutz (Impr.) v.68, n.2, 2009.
SILVA-LUZ, C.L. \& PIRANI, J.R. 2010. Anacardiaceae. In R.C. Forzza et al. (org.) Catálogo de plantas e fungos do Brasil. Rio de Janeiro: Jardim Botânico do Rio de Janeiro, v. 1, p. 599602.

SILVEIRA, M. O. O. preparo de amostras biológicas para microscopia de varredura eletrônica. In: W. de Souza (ed.). Manual sobre técnicas básicas em microscopia eletrônica de varredura, Técnicas básicas. Sociedade Brasileira de Microscopia Eletrônica, v. 1, p. 172-82. 1989.

SOARES, E. B. Avaliação de Genótipos de Cajazeira (Spondias Mombin L.): Caracterização FísicoQuímica dos Frutos e Repetibilidade e Caracteres Morfoagronômicos. Teresina, PI. Dissertação de Mestrado. Universidade Federal do Piauí, UFPI, 2005.

SOARES, E. B.; GOMES, R. L. F.; CARNEIRO, J. G. M.; NASCIMENTO, F. N.; SILVA, I. C. V.; COSTA, J. C. L. Caracterização física e química de frutos de cajazeira. Revista Brasileira de Fruticultura, v.28, n.3, p. 518-519, 2006.

SOBRINHO, I. S. B. Propriedades nutricionais e funcionais de resíduos de abacaxi, acerola e cajá oriundos da indústria produtora de polpas. Dissertação de Mestrado em Ciências Ambientais - Universidade Estadual do Sudoeste da Bahia UESB, Bahia, 2014.

SOUZA, F. X. Crescimento e desenvolvimento de clones enxertados de cajazeira na Chapada do Apodí, Ceará. Fortaleza, CE. Tese Doutorado em Agronomia. Universidade Federal do Ceará, UFC, 2005.

TIBURSKI, J.H., ROSENTHAL, A., DELIZA, R., GODOY, R.L.O., PACHECO, S. Nutritional properties of yellow mombin (Spondias mombin L.) pulp. Food Res. Int. 44,2326-2331, 2011.

VANDERZANT, C.; SPLITTSTOESSER, D. F. Compendium of methods for the microbiological examination of foods. 3th. ed. Washington: American Public Health Association, 1992. 1219 p.

VAN SOEST, P.J. Development of a comprehensive system of feed analysis and its application to forage. Journal of Animal Science, v.26, n.1, p.119-128. 1967. 